SLAC-PUB-12104

September 2006

\title{
ON THE RF DISTRIBUTION SYSTEM FOR A SET OF STANDING-WAVE ACCELERATOR STRUCTURES
}

\author{
Sami G. Tantawi \\ $S L A C$
}

(Dated: September 13, 2006)

\begin{abstract}
In this paper, we study the RF feeding system for a set of standing-wave accelerator structures. To avoid the initial reflections produced by the structures, sometimes these structures are fed in pairs through a four-port 3-dB Hybrid. We present an extension to this system for an arbitrary number of accelerator structures and show it is always possible to cancel the reflection back to the source. The necessary and sufficient condition for this to happen depends only on the spacing between accelerator structures. In this system, the structures are not fed in a binary hierarchal system, rather in series with a set of directional couplers designed to bleed off an equal amount of power to each accelerator structure in the set. We study the sensitivity of such a system to errors resulting from the differences in accelerator structures spacing. We also study the sensitivity of the system to component imperfections, such as the finite directivity of the directional couplers, and the residual reflections from the loads that are attached to these couplers. We also study the system under fault conditions, such as a breakdown in an accelerator structure or a feed waveguide.
\end{abstract}

Submitted to Physical Review Special Topics, Accelerators and Beams

Work supported by Department of Energy contract DE-AC02-76SF00515 


\section{INTRODUCTION}

Unlike traveling-wave accelerator structures, standing-wave accelerator structures gives a high-reflected signal at the beginning of the input pulse. This is usually managed by using a microwave circulator at the input port of the structure. An example of this RF system is presented in the TESLA Design Report [1]. It is also possible to feed two accelerator structures together through a 3-dB hybrid. If the distance between one of the proper ports of the 3-dB hybrid and the first accelerator structure is equal to the distance between the second accelerator structure and the second proper 3-dB hybrid port, the reflected signal is directed to the fourth port of the hybrid where it is absorbed in a load. This isolates the structures from the RF source. This technique was used for feeding SLAC's X-band standing-wave accelerator structures [2]. Here it is shown that feeding a set of standingwave accelerator structures with a chain of directional couplers can be arranged so that there is no reflection back to the source. Also the structures are essentially decoupled from each other. The system response under fault conditions due to RF breakdown in the

feeding waveguide or accelerator structures is studied, as well as the system response and the coupling between accelerator structures due to the finite directivities of the directional couplers. This type of RF distribution system is cost effective in long linacs containing many accelerator structures, such as the ILC [3], because it eliminates circulators and simplifies the RF waveguide distribution system.

\section{SYSTEM ANALYSIS}

Consider the system shown in Fig. 1. In this system, a set of $n$ standing-wave accelerator structures are fed by one waveguide running parallel to the structures. The waveguide is interrupted by a series of $n-1$ directional couplers that bleeds off some of the RF power in the waveguide to feed the accelerator structures. The reflected signal from the accelerator structures is then partially fed to the loads attached to the directional couplers and depending on their relative phases some power is reflected to the source and some is lost in the loads. To the extent of the finite directivity of the directional couplers, there should be no power coupling from one accelerator structure to another due to the reflected power. Clearly the last directional coupler (i.e., the $n-1$ coupler) has to be a 3-dB Hybrid 


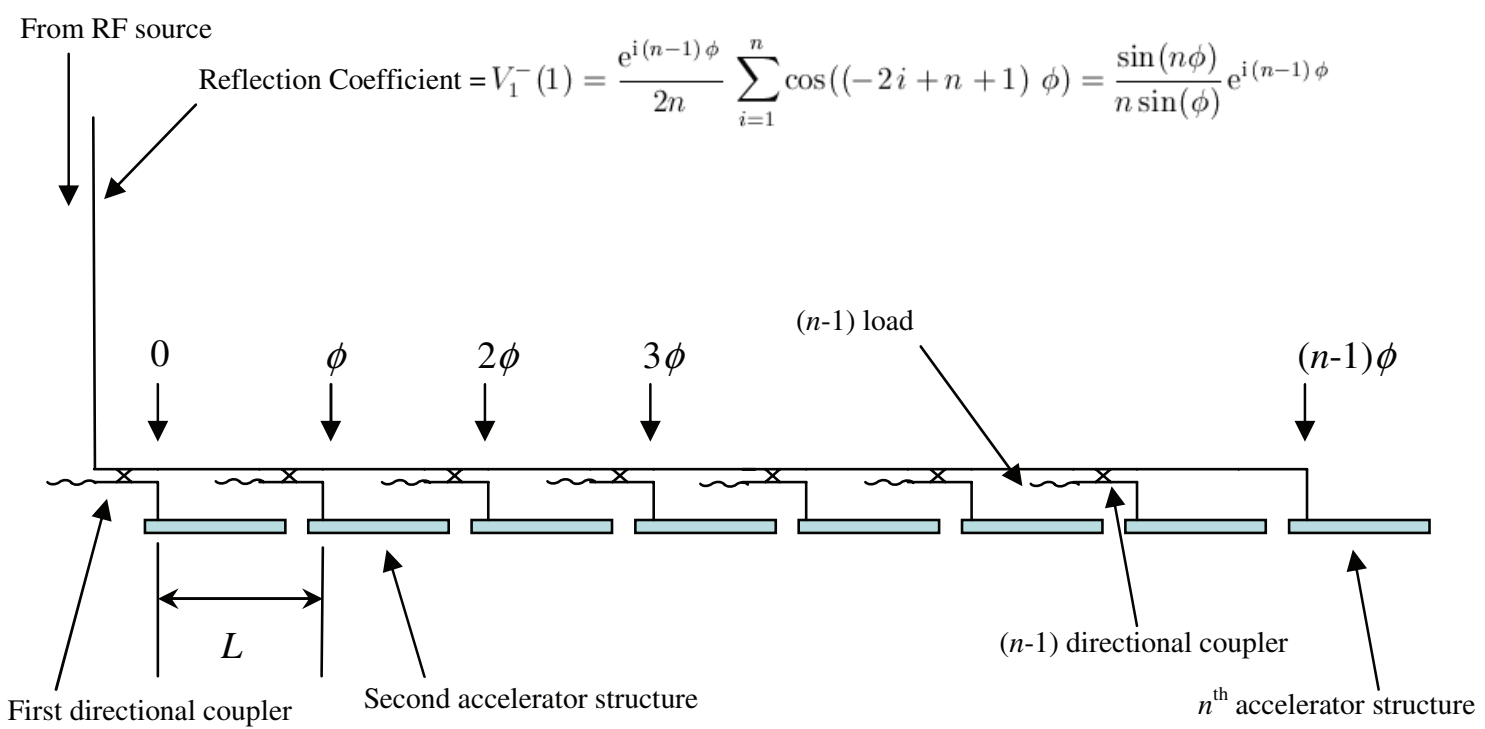

Figure 1: A chain of directional couplers feeding a set of standing-wave accelerator structures.

to split the power equally between the $n$ and the $n-1$ accelerator structure. The directional coupler before that, the $n-2$ coupler, has to couple one-third of the incident power to the structure and passes the remaining two-thirds of the power to the next coupler, etc. A general expression for the scattering matrix of the $i^{\text {th }}$ four-port directional coupler that guarantees unitarity and reciprocity, is then given by:

$$
S c(i)=\left(\begin{array}{cccc}
0 & \frac{1}{\sqrt{1-i+n}} & 0 & \sqrt{1+\frac{1}{-1+i-n}} \\
\frac{1}{\sqrt{1-i+n}} & 0 & \sqrt{1+\frac{1}{-1+i-n}} & 0 \\
0 & \sqrt{1+\frac{1}{-1+i-n}} & 0 & -\frac{1}{\sqrt{1-i+n}} \\
\sqrt{1+\frac{1}{-1+i-n}} & 0 & -\frac{1}{\sqrt{1-i+n}} & 0
\end{array}\right) .
$$

The port numbers are shown in Figure 2. To guarantee acceleration for a speed of light particle, the RF phase difference, $\phi$, between two successive accelerator structures depends on the spacing $L$ between the structures and is given by:

$$
\phi=-\frac{L \omega}{c}+2 j \pi, j \in \mathbb{Z}
$$

where $\omega$ is the operating angular frequency and $c$ is the speed of light in free space. To adjust this phase both the spacing between directional couplers, and the length of the arm between the directional coupler and the accelerator structure are used. Note the phase angle for the space between the $i^{t h}$ coupler and $\left(i^{t h}+1\right)$ coupler by $\theta(i)$. 


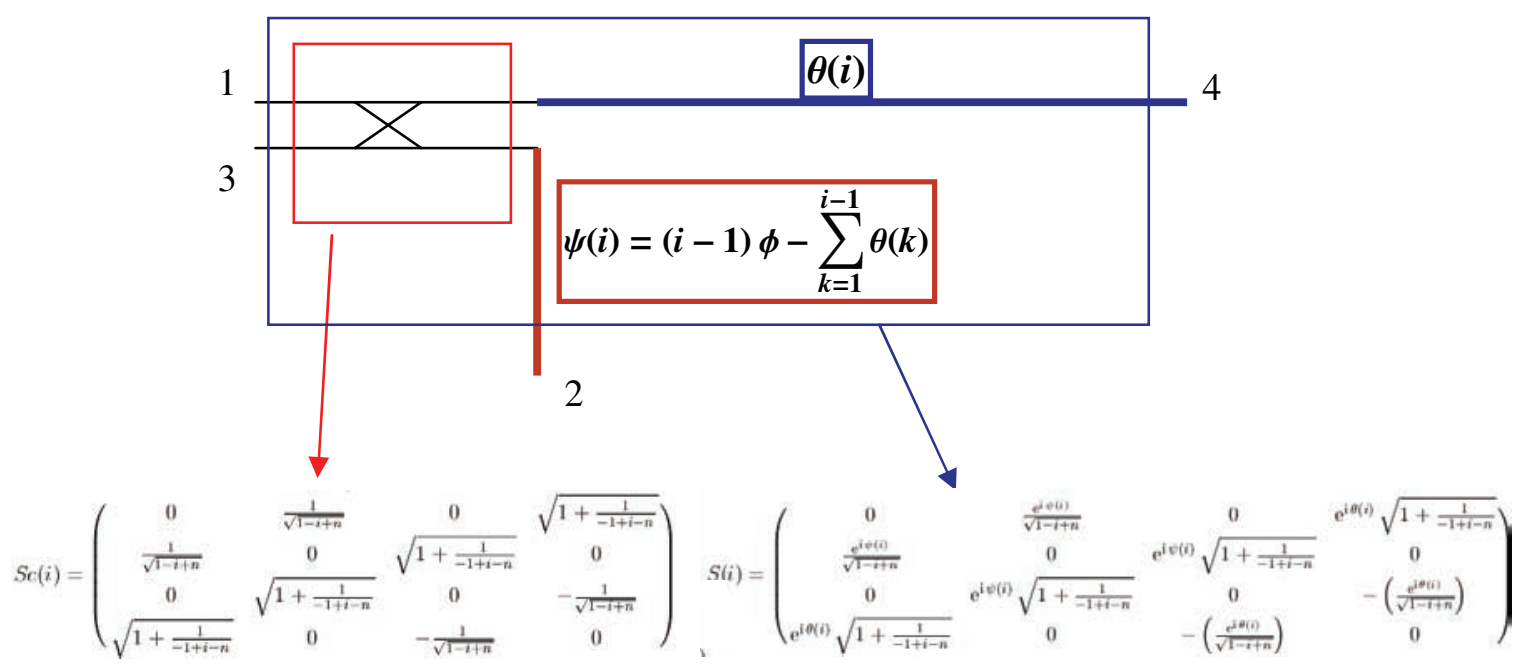

Figure 2: The $i^{\text {th }}$ directional coupler representation.

In order to satisfy Eq.(2) the phase angle $\psi(i)$ produced by the arm connecting the $i^{\text {th }}$ directional coupler to the $i^{\text {th }}$ accelerator structure has to have the following form:

$$
\psi(i)=(i-1) \phi-\sum_{k=1}^{i-1} \theta(k) .
$$

Given the phase lengths at the fourth and second ports of the $i^{\text {th }}$ directional coupler, one can define a new scattering matrix for the directional couplers, see Figure 2. The ports of this scattering matrix are defined as follows:

- Port 1 is the input of the coupler.

- Port 2 is located at the input of the $i^{\text {th }}$ accelerator structure.

- Port 3 is connected to a matched load.

- Port 4 is located at the input of the $\left(i^{t h}+1\right)$ coupler.

This scattering matrix is given by:

$$
S(i)=\left(\begin{array}{cccc}
0 & \frac{\mathrm{e}^{\mathrm{i} \psi(i)}}{\sqrt{1-i+n}} & 0 & \mathrm{e}^{\mathrm{i} \theta(i)} \sqrt{1+\frac{1}{-1+i-n}} \\
\frac{\mathrm{e}^{\mathrm{i} \psi(i)}}{\sqrt{1-i+n}} & 0 & \mathrm{e}^{\mathrm{i} \psi(i)} \sqrt{1+\frac{1}{-1+i-n}} & 0 \\
0 & \mathrm{e}^{\mathrm{i} \psi(i)} \sqrt{1+\frac{1}{-1+i-n}} & 0 & -\left(\frac{\mathrm{e}^{\mathrm{i} \theta(i)}}{\sqrt{1-i+n}}\right) \\
\mathrm{e}^{\mathrm{i} \theta(i)} \sqrt{1+\frac{1}{-1+i-n}} & 0 & -\left(\frac{\mathrm{e}^{\mathrm{i} \theta(i)}}{\sqrt{1-i+n}}\right) & 0
\end{array}\right) .
$$


Assuming all standing-wave accelerator structures are identical, one can calculate the reflected power to the source. During the beginning of the charging phase (i.e., at a time $t<<t_{f}$; where $t_{f}$ is filling time of the accelerator structure) most of the power is being reflected from the accelerator structure. One can then consider the worst case scenario, where all the power is being reflected simultaneously from all accelerator structures. The input vector to the $i^{\text {th }}$ scattering matrix is then given by:

$$
V^{+}(i)=\left(\begin{array}{c}
\mathrm{e}^{\mathrm{i}\left(\sum_{k=1}^{-1+i} \theta(k)\right)} \sqrt{\frac{1-i+n}{n}} \\
\frac{\mathrm{e}^{\mathrm{i}(-1+i) \phi}}{\sqrt{n}} \\
0 \\
V_{4}^{+}(i)
\end{array}\right) .
$$

The first component of that vector represents the input power to the coupler after the signal has passed through the previous $i-1$ couplers. The second component represents the reflected signal from the $i^{\text {th }}$ structure. The third components represent the reflection from the load attached to the coupler and we assumed a matched load. The fourth component $V_{4}^{+}(i)$ represents the reflected signal from all the remaining accelerator structures after being scattered through the remaining directional couplers. The scattered fields from the $i^{t h}$ directional coupler is given by:

$$
V^{-}(i)=S(i) V^{+}(i)
$$

The scattered field from Port 1 of the $i^{\text {th }}$ directional coupler is the input to Port 4 of the $i-1$ coupler. For example:

$$
V_{4}^{+}(i-1)=V_{1}^{-}(i) .
$$

For the last directional coupler in the chain, the $n-1$ coupler, $V_{4}^{+}$is simply the reflection from the last, the $n^{\text {th }}$, accelerator structure and is given by:

$$
V_{4}^{+}(n-1)=\mathrm{e}^{2 \mathrm{i}(n-1) \phi-\mathrm{i} \sum_{k=1}^{-1+n} \theta(k)} \frac{1}{\sqrt{n}} .
$$

Using Eqs.(4) through (8) one can calculate the reflected signal to the source. By induction, a general expression for that coefficient can be calculated and is given by:

$$
V_{1}^{-}(1)=\frac{\mathrm{e}^{\mathrm{i}(n-1) \phi}}{2 n} \sum_{i=1}^{n} \cos ((-2 i+n+1) \phi)=\frac{\sin (n \phi)}{n \sin (\phi)} \mathrm{e}^{\mathrm{i}(n-1) \phi} .
$$

$\mathrm{Eq}(9)$ is the fundamental result of this work. It shows that the reflection to the source depends only on the spacing between accelerator structures (i.e., the angle $\phi$ ). Also, it 


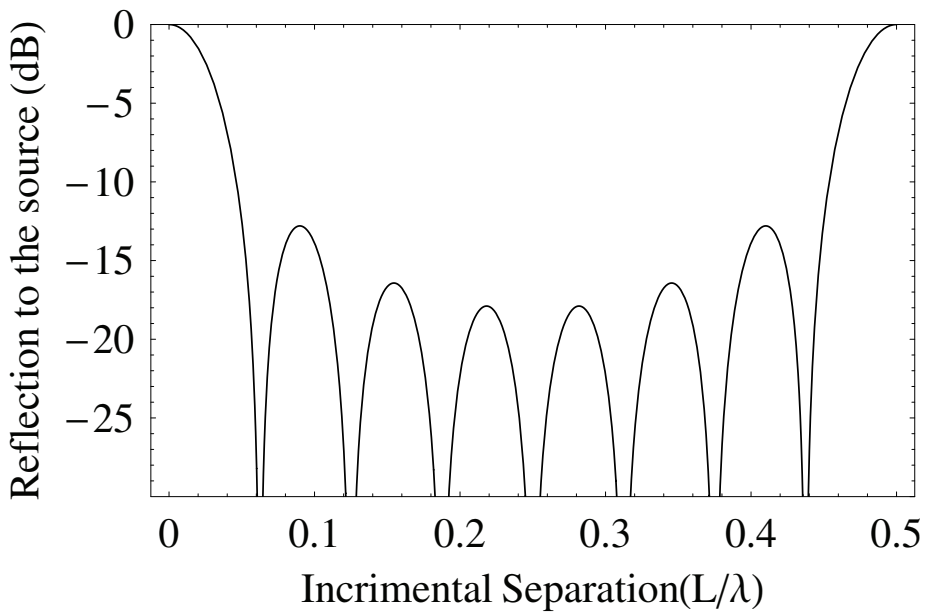

Figure 3: System response as seen from Port 1 of the first directional coupler. The system contains eight accelerator structures and seven directional couplers

shows that it is always possible to choose this spacing to cancel the reflection completely to the sources; this happens at $\phi=i \pi / n, i \neq j n, i, j \in \mathrm{Z}$. Using $\operatorname{Eq}(2)$, this spacing is then given by:

$$
L(i)=j \lambda-\frac{i \lambda}{2 n}, L \neq k \lambda / 2, i, j, k \in \mathrm{Z}
$$

$\mathrm{Eq}(10)$ is plotted in Figure 3 for the case of eight accelerator structures, $(n=8)$. Several comments on this graph are in order. The envelop of the reflection coefficient has a minimum at $L=i \lambda / 2+\lambda / 4, i \in \mathrm{Z}$. One can show that this minimum is equal to $1 / n$. If one chooses the separation between accelerator structure near this minimum, the resulting system would not be very sensitive to the tolerance on this separation (i.e., one need not hit exactly the septation that nulls the refection coefficient). This gives the system designer more freedom to choose the distance between accelerator structures.

Some of the reflected power from the structures are absorbed by the loads that are attached to the directional couplers. For $n$ structures having a $\phi$ phase advance between each two consecutive structures, an explicit expression for the power dissipated in $j^{\text {th }}$ load is:

$$
P(j, \phi, n)=\frac{\sin ^{2}((j-n) \phi) \csc ^{2}(\phi)}{n(n-j)(n-j+1)}-\frac{\csc (\phi) \sin ((1-2(j-n)) \phi)}{n(n-j+1)}+\frac{1}{n} .
$$

For the spacing between structures that makes the reflection coefficient identically zero, the reflected power from the accelerator structures is distributed among the loads. In this case, 


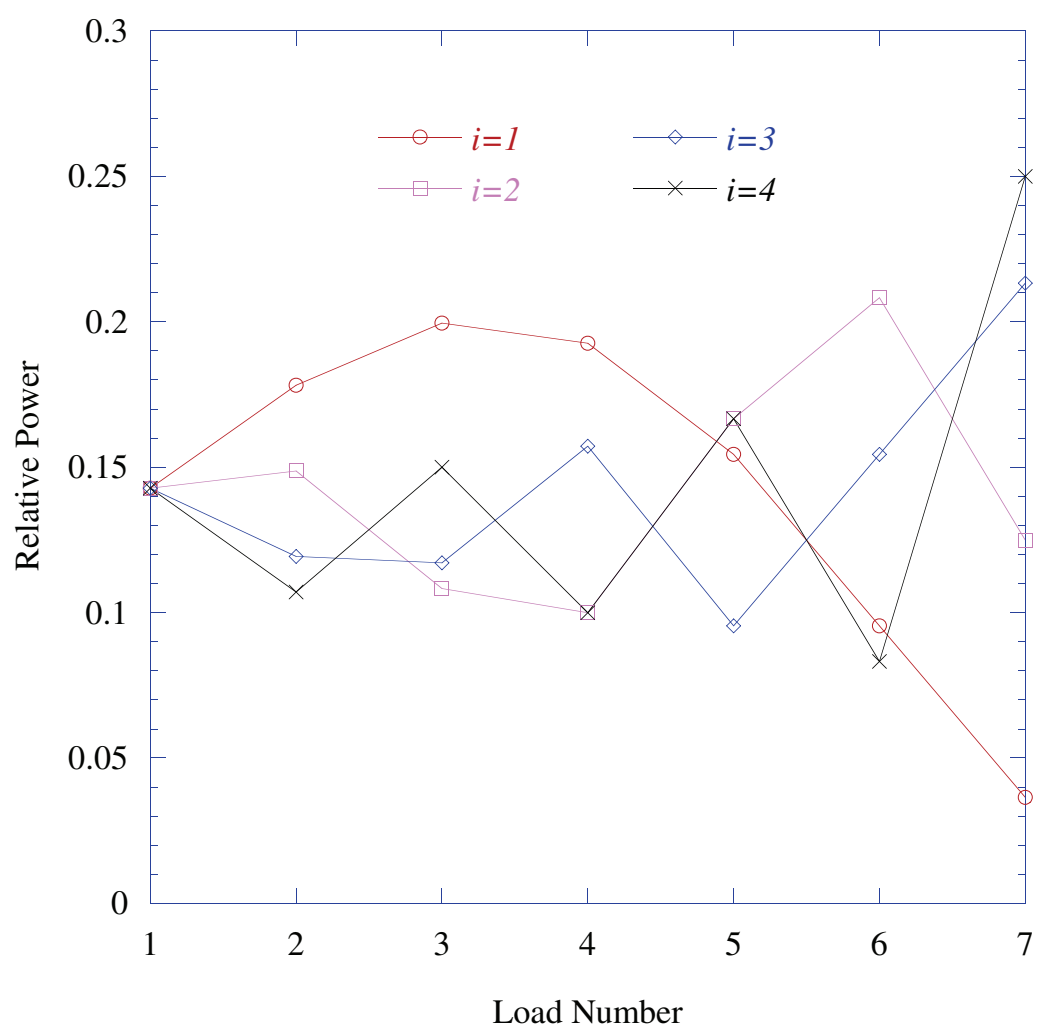

Figure 4: Power distribution over the loads for a system satisfying Eq(10). The number of directional couplers and loads is seven and the number of accelerator structures is eight.

for a system with $n$ structures separated by $L(i), \mathrm{Eq}(11)$ reduces to:

$$
P(j, i, n)=\frac{\sin ^{2}\left(\frac{i(n-j) \pi}{n}\right) / \sin \left(\frac{i \pi}{n}\right)-(n-j) \sin \left(\frac{i(2(n-j)+1) \pi}{n}\right)}{(n-j)(n-j+1) n \sin \left(\frac{i \pi}{n}\right)}+\frac{1}{n} .
$$

Note that $\sum_{j=1}^{n-1} P(j, i, n)=1$. Because $P(j, i, n)$ is an even function of $i$, there is only $\lfloor n / 2\rfloor$ possible distributions. Figure 4 shows a plot of the power distributions among the loads for an eight-structure system for different structure separation $L(i), i=1,2,3,4$.

\section{SYSTEM RESPONSE UNDER FAULT CONDITIONS}

\section{A. Residual Reflection due to Structure Failure}

Now consider what happens if one of the structures fails. The following calculates two cases. First, if the structure or its fundamental mode coupler starts to arc, and instead of reflecting the power the power gets absorbed by the arc. It is assumed, for simplicity, the 
extreme case of the power being absorbed completely by the $i^{\text {th }}$ structure. Second, consider the case of a structures or coupler failure long after all the structures are filled with energy and essentially do not reflect any power back to the source. Also, for simplicity, consider the extreme case of unity reflection coefficient from the $i^{\text {th }}$ structure, while the rest of the structures are essentially matched.

For the first case, the residual reflection coefficient is:

$$
R_{\text {res }}=V_{1}^{-}(1)=\frac{\mathrm{e}^{\mathrm{i}(n-1) \phi} \csc (\phi) \sin (n \phi)-\mathrm{e}^{2 \mathrm{i}(i-1) \phi}}{n}
$$

If $\phi$ satisfies the spacing conditions given by $\mathrm{Eq}(10)$ then the magnitude of $\left|R_{\text {res }}\right|=1 / n$. In the second case, where only one of the accelerator structures is reflecting the incident power the residual reflection is also $\left|R_{\text {res }}\right|=1 / n$. This is obvious since the magnitude of the coupling between the input port and any output port is $1 / \sqrt{n}$.

\section{B. Residual Reflection and Coupling Between Structures due to Components Er-} rors

Consider the residual reflection and coupling between structures due to the finite directivity of the directional couplers and mismatches at the loads attached to them. The construction of most directional coupler is such that the device has a set of reflection sym-

metries. Hence, at some reference planes that are chosen to reveal these symmetries, the scattering matrix of an imperfect directional coupler should take the following form [4]:

$$
S=\left(\begin{array}{llll}
\alpha & \beta & \gamma & \zeta \\
\beta & \alpha & \zeta & \gamma \\
\gamma & \zeta & \alpha & \beta \\
\zeta & \gamma & \beta & \alpha
\end{array}\right)
$$

Imposing unitarity to this form and shifting the reference planes of Ports 3 and 4 by $-\pi / 2$, one can write a an expression for the scattering matrix of an imperfect directional coupler that is compatible with $\operatorname{Eq}(1)$ :

$$
S=\left(\begin{array}{cccc}
-\alpha & \beta & \gamma & \zeta \\
\beta & -\alpha & \zeta & \gamma \\
\gamma & \zeta & \alpha & -\beta \\
\zeta & \gamma & -\beta & \alpha
\end{array}\right)
$$




$$
\begin{aligned}
& \alpha=\delta_{i} \sqrt{\frac{\delta_{i}^{2}+i-n}{(i-n-1)\left(\delta_{i}^{2}+1\right)}} ; \\
& \beta=\frac{1}{\sqrt{n-i+1}} ; \\
& \gamma=\frac{\delta_{i}}{\sqrt{n-i+1}} ; \\
& \zeta=\frac{\sqrt{\frac{i^{2}+\left(\delta_{i}^{2}-2 n-1\right) i+n^{2}-n \delta_{i}^{2}-\delta_{i}^{2}+n}{\delta_{i}^{2}+1}}}{n-i+1} ;
\end{aligned}
$$

where the directivity of the coupler $d_{i}=-20 \log \left(\left|\delta_{i}\right|\right), \delta_{i} \in \mathrm{R},\left|\delta_{i}\right| \ll 1$.

Now, we introduce a load at the third arm of the above four-port network. The load has a residual reflection coefficient $\epsilon_{i}, \epsilon_{i} \in \mathbb{C},\left|\epsilon_{i}\right| \ll 1$. The resultant three-port network has a scattering matrix which, of course, is not unitary. We expand this matrix in orders of $\delta_{i}$ and $\epsilon_{i}$ and keep only first order terms. Then the three-port network is represented by:

$$
S_{3}(i)=\left(\begin{array}{lll}
-\sqrt{1+\frac{1}{i-n-1}} \delta_{i} & \frac{1}{\sqrt{-i+n+1}} & \sqrt{1+\frac{1}{i-n-1}} \\
\frac{1}{\sqrt{-i+n+1}} & \sqrt{1+\frac{1}{i-n-1}}\left(\sqrt{1+\frac{1}{i-n-1}} \epsilon_{i}-\delta_{i}\right) & \frac{\delta_{i}-\sqrt{1+\frac{1}{i-n-1}}}{\sqrt{-i+n+1}} \\
\sqrt{1+\frac{1}{i-n-1}} & \frac{\delta_{i}-\sqrt{1+\frac{1}{i-n-1}}}{\sqrt{-i+n+1}} & \sqrt{1+\frac{1}{i-n-1}} \delta_{i}+\frac{\epsilon_{i}}{-i+n+1}
\end{array}\right) .
$$

With reference to Figure 2, we move reference planes of Ports 2 and 3 with an angle $\psi(i)$, $\theta(i)$ respectively. This is similar to the transition from $\mathrm{Eq}(1)$ to $\mathrm{Eq}(4)$. Having an expression for the the three-port scattering matrix for each coupler $i$ allows us to cascade all of them together [5] to get the total $(n+1) \times(n+1)$ scattering matrix $S$ of the system. We now list the results of this procedure that is correct only to first order in $\delta_{i}$ and $\epsilon_{i}$.

As expected the coupling between the first port, Port 1 and the remaining $n$ ports is given by:

$$
S_{1 i}=\frac{\mathrm{e}^{\mathrm{i}(i-2) \phi}}{\sqrt{n}}, 2 \leq i \leq n+1 .
$$

The intrinsic reflection coefficient of the system is now finite and is given by:

$$
S_{11}=-\sum_{j=1}^{n-1} \mathrm{e}^{2 \mathrm{i} \sum_{k=1}^{j-1} \theta(k)} \frac{\sqrt{(n-j)(n-j+1)}}{n} \delta_{j} .
$$

The coupling between feeding ports is given by $S_{i j}=S_{j i}$ that represent the coupling between the port that feed accelerator $j-1$ and accelerator $i-1$ and is given by:

$$
S_{j i}=\mathrm{e}^{\mathrm{i}(i+j-4) \phi}\left(\mathrm{e}^{-2 \mathrm{i} \sum_{l=1}^{j-2} \theta(l)}\left(\frac{\delta_{j-1}}{\sqrt{(-j+n+1)(-j+n+2)}}-\frac{\epsilon_{j-1}}{-j+n+2}\right)+\right.
$$




$$
\left.\sum_{k=1}^{j-2} \mathrm{e}^{-2 \mathrm{i} \sum_{l=1}^{k-1} \theta(l)}\left(\frac{\delta_{k}+\epsilon_{k}}{\sqrt{(n-k)(-k+n+1)}}+\right)\right), 2 \leq j \leq n, i>j .
$$

The accelerator structure feeds are also mismatched and have a residual reflection coefficient and is given by:

$$
\begin{array}{r}
S_{i i}=\mathrm{e}^{2 \mathrm{i}(i-2) \phi}\left(\mathrm{e}^{-2 \mathrm{i} \sum_{l=1}^{i-2} \theta(l)}\left(\frac{(n-i)}{-i+n+1} \epsilon_{i-1}-\sqrt{\frac{n-i}{-i+n+1}} \delta_{i-1}\right)+\right. \\
\left.\sum_{j=1}^{i-2} \mathrm{e}^{-2 \mathrm{i} \sum_{l=1}^{j-1} \theta(l)}\left((n-i)^{|i-j-2|} \epsilon_{j}(n-i+1)^{j-i}+(n-i)^{\left|i-j-\frac{3}{2}\right|} \delta_{j}(n-i+1)^{\left(j-i+\frac{1}{2}\right)}\right)\right), \\
2 \leq i \leq n .
\end{array}
$$

Finally, the reflection seen by the $n^{\text {th }}$ accelerator structure is given by:

$$
S_{(n+1)(n+1)}=\mathrm{e}^{2 \mathrm{i}(n-1) \phi} \sum_{j=1}^{n-1} \mathrm{e}^{-2 \mathrm{i} \sum_{l=1}^{j-1} \theta(l)}\left(\frac{\delta_{j}}{\sqrt{(n-j)(n-j+1)}}+\frac{\epsilon_{j}}{(n-j)(n-j+1)}\right) .
$$

Eqs. (21) to (25) present all matrix elements for the scattering matrix representing the distribution system.

Assuming that all the directivities of the couplers are equal; $\delta_{j}=\delta$, the residual reflection coefficient, $\mathrm{Eq}(22)$, can have an upper limit when all the phases in the series are the same (i.e., $\theta(k)-\theta(1)=i \pi, i, k \in \mathrm{Z})$. This upper limit is $\approx n \delta / 2$. Up to this point, there is no reason to specify the choice of $\theta(k)$. It is obvious that one can minimize or at least reduce the residual reflection by scrambling the phases of the summation in $\operatorname{Eq}(22)$.

The same of course can be said about the coupling between accelerator structures; the phases in the summation term can be used to minimize the coupling. However, in this case one can also tune each coupler individually by adjusting the load reflection coefficient, $\epsilon_{k}$ with respect to $\delta_{k}$. This is accomplished experimentally by perturbing the the waveguide connecting the load to the coupler. Finally, the reflection coefficient seen by each structure benefits from this tuning procedure as well, but it can be taken into account when tuning the coupling of each individual accelerator structure.

\section{CONCLUSION}

The RF feeding system for a set of standing-wave accelerator structures was presented. The system comprises a chain of directional coupler connected in series. Each coupler bleeds 
off an equal amount of power, and hence, their coupling is tapered along the chain. We also presented a set of expressions that enables system designers to estimate system parameters and errors. The fundamental results of this work is summarized in the following few points:

1. The reflected signal to the source depends only on the spacing between accelerator structure.

2. By appropriate choice of the spacing between accelerator structures, the reflection to the source from the system could be eliminated, see $\operatorname{Eq}(10)$. Hence, we eliminate the need for using circulators in such a system.

3. The system has the advantage of low sensitivity to the system parameters as the number of couplers in the chain is increased. The reflection to the source is least sensitive near spacing $L=i \lambda / 2+\lambda / 4, i \in \mathrm{Z}$.

4. At fault conditions the reflected power to the source is $\sim 1 / n^{2}$.

5. However, the value of an increased number of feeds starts to diminish at around 8 to 10 feeds. At the same time, errors due to components imperfection start to increase. These errors are minimized by tuning individual couplers with respect to the attached loads and also by adjusting the spacing between couplers.

\section{ACKNOWLEDGEMENT}

We would like to thank Prof. Ronald Ruth of SLAC, Dr. Dieter Proch, Dr. Stefan Choroba, Dr. Valery Katalev, Dr. Wolf-Dietrich Möller, and Dr. Jacek Sekutowicz of DESY for very useful discussions.

[1] R. Brinkman, K. Flottman, J. Roßbach, P. Schmusser, N. Walker, and H. Weise(eds.), TESLA Technical Design Report, Tech. Rep., DESY, Hamborg, Germany (2001), TESLA Technical Design Report, http://tesla.desy.de/new_pages/TDR_CD/start.html.

[2] in V. A. Dolgashev, C. Adolphsen, D. Burke, G. Bowden, R. Jones, J. Lewandowski, Z. Li, R. Loewen, R. Miller, C. Ng, C. Pearson, R. Ruth, et al.Proc. of the Particle Accelerator Conference (PAC 03) (2003), pp. 1264-1266. 
[3] First ILC Workshop-Towards an International Design of a Linear Collider (KEK, Tsukuba, Japan, 2004), http://lcdev.kek.jp/ILCWS/.

[4] C. G. Montgomery, R. H. Dicke, and E. M. Purcell, Principles of Microwave Circuits (Boston Technical Lithographers, Inc., 5 Bryant Road, Lexington, Massachusetts, USA, 1963)MIT Radiation Laboratory Series of vol. 8, section12.17, p. 437, first ed.

[5] J. Neilson, P. Latham, M. Caplan, and W. Lawson, IEEE Transactions on Microwave Theory and Techniques 37(8), 1165 (1988), see aslo Ref[3] cited therin. 